\title{
Chemical Dehydrogenation of Hydrogenated Single Layer Graphene for Reversible Electrical Conductivity
}

\author{
Prathana Paul Chowdhury, ${ }^{a}$ Tania Chatterjee, ${ }^{b}$ and Arnab Mukherjee ${ }^{\star, a}$ \\ ${ }^{a}$ Functional Materials and Devices Division, CSIR-Central Glass and Ceramic Research Institute, 196, \\ Raja S. C. Mullick Road, Jadavpur, Kolkata, West Bengal-700032, India \\ ${ }^{b}$ Advanced Mechanical and Materials Characterization Division, CSIR-Central Glass and Ceramic Research \\ Institute,196, Raja S. C. Mullick Road, Jadavpur, Kolkata, West Bengal-700032, India
}

Email: arnabm@cgcri.res.in (A. M.)

\begin{abstract}
Chemical functionalization of graphene greatly expands its potential in electronic, photovoltaics and biological applications. The band gap tunability of graphene achieved during the process of its hydrogenation and dehydrogenation can open its potential as an acceptor material in organic photovoltaic (OPV) solar cells. In this paper, we report a rapid and extensive hydrogenation of single layer graphene (SLG) by Birch reduction followed by its oxidation back to the conducting SLG. Dehydrogenation of hydrogenated SLG using 2,3-dichloro-5,6-dicyano-1,4-benzoquinone (DDQ) as an oxidising agent was performed successfully. Raman, FT-IR and UV-Vis spectra provides supporting evidence for a successful dehydrogenation reaction. A control experiment without DDQ as an oxidant confirms its active role as an oxidising agent. Magnetic moment study reveals a reversibility of ferromagnetic hydrogenated SLG to a paramagnetic dehyrdrogenated SLG. Finally, the sheet resistance measurement of pristine, hydrogenated and dehydrogenated SLG shows almost complete reversal of the electrical conductivity.
\end{abstract}

Keywords single layer graphene, reversible hydrogenation, 2,3-dichloro-5,6-dicyano-1,4-benzoquinone, magnetic moment, sheet resistance

\section{Introduction}

Graphene is a two-dimensional monolayer of $\mathrm{sp}^{2}$ carbon atoms with honey-comb like structure, ${ }^{[1,2]}$ having unique physical, ${ }^{[3]}$ mechanical and electrical properties. ${ }^{[4,5]}$ Chemical modification of graphene can tune its material properties required for a given application. ${ }^{[6-11]}$ Several types of chemical reactions have already been demonstrated on graphene. ${ }^{[12-15]}$ Out of them, chemical hydrogenation of graphene is a very popular technique. ${ }^{[16]}$ This process can transform a zero-bandgap semiconductor to a wide band gap insulator ${ }^{[17]}$ and depend on the extent of hydrogenation to a medium band gap semiconductor. ${ }^{[18-20]}$ Previous efforts at hydrogenation of graphene included physical techniques like exposing the graphene to atomic hydrogen plasma known as plasma hydrogenation. ${ }^{[13,21,22]} \mathrm{A}$ more effective and rapid route for hydrogenation of graphene is by Birch reduction, where an alkali metal is dissolved in liquid ammonia before adding graphene into it. ${ }^{[3]}$ This is followed by addition of a proton source like tert-butanol $(t-\mathrm{BuOH}) /$ methanol $(\mathrm{MeOH})$ in the reaction mixture to create a partially hydrogenated graphene. The as hydrogenated graphene can be restored back to graphene using thermal annealing technique $;^{[21]}$ however, such processes involve loss of carbon atoms from the graphene lattice leading to alternation of its properties and also are not very effective. In this paper, we report an one-step dehydrogenation of birch reduced "hydrogenated single layer graphene (SLG)" using 2,3-dichloro-5,6-dicyano-1,4-benzoquinone (DDQ) as an oxidising agent. The chemically dehydrogenated graphene has been characterized extensively and it was observed that a near pristine graphene comparable to the starting SLG can be obtained by this technique. Also, the band gap of the SLG got tuned during the hydrogenation/ dehydrogenation process and it has a potential application as an acceptor material in organic photovoltaic (OPV) solar cells replacing the presently used phenyl-C61-butyric acid methyl ester (PCBM).

\section{Experimental}

\section{Reagents}

All reagents were purchased from Sigma-Aldrich, and unless otherwise indicated, were used without further purification. DDQ was obtained from Spectrochem Pvt, Ltd. with a purity of $98 \%$. SLG was grown on copper (Cu) foil (Alfa Aesar with a purity of $99.8 \%$ ) via chemical vapor deposition (CVD) technique in a split tube furnace (with $\mathrm{Fe} \mathrm{Cr}$-Al heating element) from methane decomposition at $1000{ }^{\circ} \mathrm{C}$. Prior to the growth, the copper foil was thoroughly cleaned with acetone, glacial acetic acid, deionized water and isopropyl alcohol sequentially to get rid of any residual oxide layer on the surface. ${ }^{[23]}$

SLG grown on Cu foil was then transferred on to a quartz substrate using a solution of poly-methylmetacrylate (PMMA, 3 $w t \%)$ in anisole as described earlier, ${ }^{[24]}$ and hydrogenated using Birch reduction technique. The detailed experimental procedure for the transfer of SLG on quartz substrate is given in ESI (Figure S1).

\section{Synthesis of hydrogenated SLG by Birch reduction}

The hydrogenation of SLG was carried out following a Birch reduction procedure earlier reported on graphite nanosheets. ${ }^{[22]}$ A $250 \mathrm{~mL}$ three-neck round-bottom flask equipped with a condenser was flame-dried under vacuum, flushed with argon, and then maintained under an atmosphere of argon. A dry ice-acetone mixture $\left(-78^{\circ} \mathrm{C}\right)$ was then applied to cool the flask and the condenser. Liquid ammonia $(\sim 100 \mathrm{~mL})$ was condensed 
inside the flask, followed by the addition of one or two beads of lithium metal and single layer graphene transferred on to quartz substrate was then dropped into the mixture with gentle swirling motion given for the dissolution of lithium metal in the liquid ammonia. Liquid ammonia is used as a solvent medium for carrying out the Birch reduction. The mixture was kept for $1 \mathrm{~h}$ under argon atmosphere. The dry ice-acetone bath was then removed, and methanol $(\sim 1 \mathrm{~mL})$ was added to the reaction mixture. The reaction mixture was refluxed at the boiling temperature of liquid ammonia $\left(-33^{\circ} \mathrm{C}\right)$ and kept for $4 \mathrm{~h}$ while refilling the dry ice-cetone as needed. The ammonia in the system was then allowed to evaporate overnight. The reaction was quenched by slow addition of ethanol followed by water. The SLG substrate was taken out of the reaction mixture and washed thoroughly with water, hexane and ethanol. As obtained partially hydrogenated SLG on quartz was then dried at $80{ }^{\circ} \mathrm{C}$ in a hot air oven in ambient atmosphere for $20-25$ minutes.

The Birch reduction of SLG to yield hydrogenated graphene (graphane) is illustrated in Scheme 1.

Scheme 1 Birch reduction of SLG

$$
\mathrm{SLG}+\mathrm{Li}+\mathrm{NH}_{3} \rightarrow[\mathrm{SLG}] \mathrm{Li}^{+}
$$

\section{$[\mathrm{SLG}]^{-} \mathrm{Li}^{+}+\mathrm{CH}_{3} \mathrm{OH} \rightarrow$ Hydrogenated SLG}

\section{Synthesis of dehydrogenated SLG using DDQ}

Hydrogenated SLG on quartz was dehydrogenated using excess of DDQ and toluene refluxing at $110^{\circ} \mathrm{C}$ with continuous stirring for $24 \mathrm{~h}$. The samples were washed thoroughly using excess of toluene to remove unreacted reactants and kept for drying in an ambient atmosphere for 20-25 min.

Dehydrogenation of hydrogenated SLG using DDQ as an oxidizing agent is shown in Scheme 2.

Scheme 2 Dehydrogenation of SLG

$$
\text { Hydrogenated SLG } \frac{D D Q, \text { Tolune }}{\text { Reflux } 110^{\circ} \mathrm{C}} \rightarrow \text { Dehydrogeneted SLG }
$$

\section{Characterization}

Raman spectra were obtained using a Renishaw InVia Reflex micro Raman spectrometer with excitation by argon ion $(514 \mathrm{~nm})$ laser. The laser power was kept sufficiently low to avoid heating of the samples and the spectra were collected with a resolution of $1 \mathrm{~cm}^{-1}$. Multiple spectra (3-5) were obtained, normalized to the $G$ band, and averaged out to present a comprehensive overview of the material. Fourier transform infrared (FT-IR) spectra of the samples were recorded on a Perkin Elmer FTIR Spectrometer in reflectance mode. UV-Visible absorption spectra were recorded using a Cary 50, Varian Inc spectrometer. Two probe conductivity (current-voltage) measurements were taken on a Keithley 4200 SCS performed on SLG transferred onto quartz substrate. Magnetism of the sample was studied using SQUID Magnetometer at room temperature on quartz transferred SLG.

\section{Results and Discussion}

Raman spectra of the SLG on Cu foil, SLG transferred onto quartz substrate, hydrogenated and dehydrogenated SLG on quartz is shown in Figure 1. The large 2D band observed in the SLG on copper foil and SLG transferred onto quartz substrate is indicative of the high quality single layer graphene with very less defects as is observed by a very small " $D$ " or defect band at $1320 \mathrm{~cm}^{-1}$ in the pristine SLG. A miniscule D band appearing in the transferred SLG is due to the defects that get incurred on to this single atom thick graphene layer during the process of transfer. The huge $D$ band observed at $1320 \mathrm{~cm}^{-1}$ in the hydrogenated SLG is indicative of the chemical disruption of the $\mathrm{sp}^{2}$-hybridized carbon network that results from the covalent attachment of hydrogen atoms. In addition, a decrease and broadening of the 2D band (also called the G' band) at 2680 $\mathrm{cm}^{-1}$ and an increase of the (D+D') combination band at 2890 $\mathrm{cm}^{-1}$ were also observed, which confirms the high degree of hydrogenation. ${ }^{[2]}$ The reoccurrence of the $2 \mathrm{D}$ band, disappearance of the D+D' combination band and considerable decrease of the $D$ band in de-hydrogenated SLG confirms the dehydrogenation of SLG. A control experiment of dehydrogenation without using DDQ was performed keeping all other conditions same. The Raman spectrum of the final product shows the presence of $D+D$ ' band and also presence of $D$ band indicating that almost no dehydrogenation has occurred as shown in ESI (Figure S2).

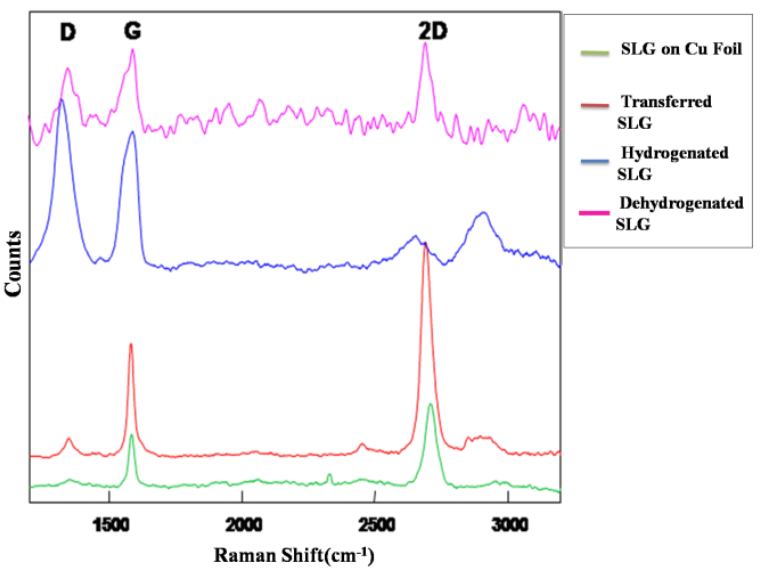

Figure 1 Raman Spectra of SLG, transferred SLG, hydrogenated and dehydrogenated SLG.

FT-IR spectra of the SLG, hydrogenated SLG and dehydrogenated SLG are shown in Figure 2. The hydrogenated SLG exhibits a strong peak at $\sim 2850$ and $\sim 2910 \mathrm{~cm}^{-1}$, indicative of the aliphatic $\mathrm{C}-\mathrm{H}$ stretching mode. ${ }^{[23]}$ No peak was observed in this region for the pristine SLG and again after dehydrogenation. The peaks almost disappeared confirming the success of the dehydrogenation reaction.

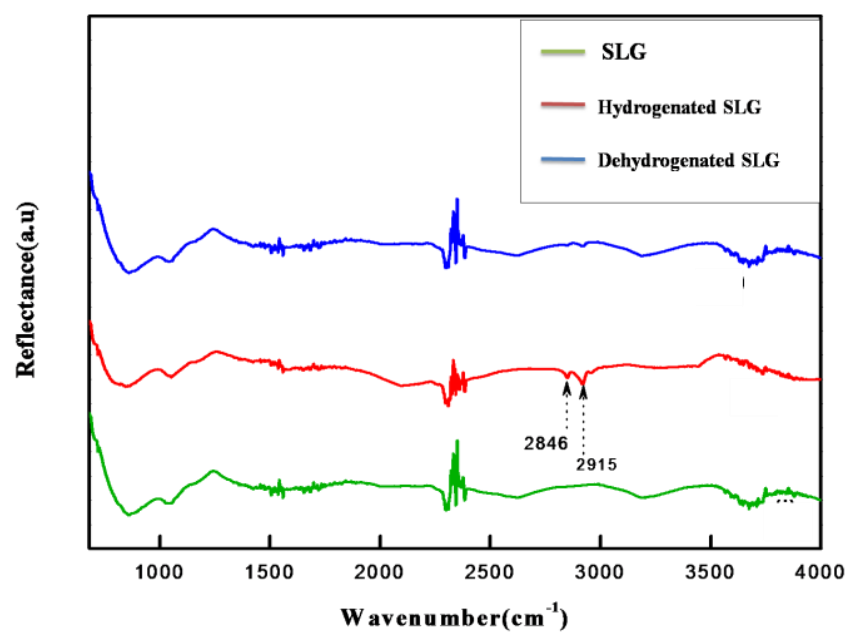

Figure 2 FT-IR spectra of SLG, hydrogenated and dehydrogenated SLG.

The band gap of the hydrogenated SLG was determined to be $\sim 2.5 \mathrm{eV}$ using UV-Vis spectroscopy with the help of plots 
that were plotted using Tauc's equation. ${ }^{[26,27]}$ The increase in band gap from starting SLG $(\sim 1.56 \mathrm{eV})$ emphasizes the high degree of hydrogenation, similarly, the reversal of the band gap to $\sim 1.7 \mathrm{eV}$ supports the process of dehydrogenation as shown in Figure 3. Band gap in the otherwise zero band gap pristine SLG originates due to the presence of defects incurred in it during the transfer as it was observed from the appearance of "D" band in the Raman spectra of the transferred SLG (Figure 1). Sharp changes in the UV spectrum at $\sim 3.5 \mathrm{eV}(\sim 350 \mathrm{~nm})$ results from the lamp change in the spectrophotometer. The original UV-Vis spectra of SLG, hydrogenated SLG and dehydrogenated SLG are shown in ESI (Figure S3).

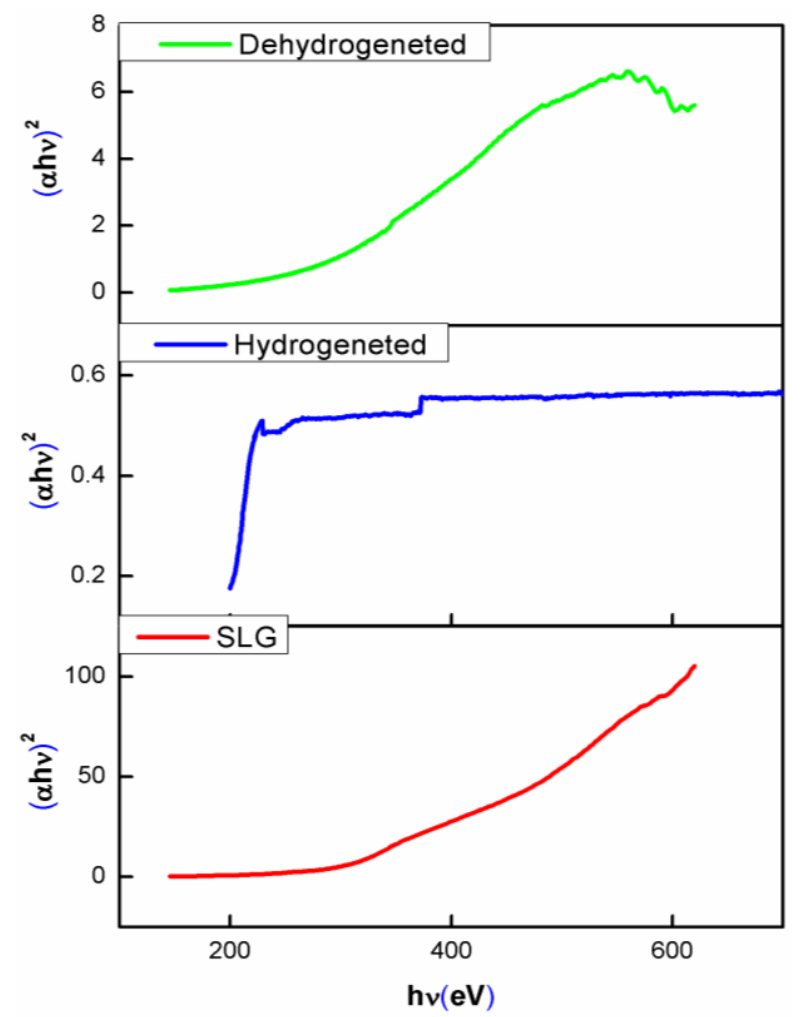

Figure 3 Band gap study of the SLG, hydrogenated and dehyrdrogenated graphene measured with UV-Vis spectroscopy and then plotted using Tauc's equation.

Magnetic moment study of the hydrogenated SLG showed a clear transformation from paramagnetic nature of the pristine SLG to ferromagnetic behavior. Partial hydrogenation breaks down the delocalized $\pi$ bonding network of graphene, leaving the electrons in the non-hydrogenated carbon atoms localized and unpaired. The magnetic moments at these sites couple ferromagnetically at room temperature giving rise to an infinite magnetic sheet with structural integrity and magnetic homogeneity. ${ }^{[28]}$ However, with dehydrogenation such unpaired electrons and dangling bonds are removed and the extended $\pi$ bonding network of the starting graphene is recovered and therefore the paramagnetic nature of the pristine SLG is revived back (Figure 4). M-H data for starting pristine SLG is shown in ESI (Figure S4).

FESEM image of SLG on copper foil is shown in Figure 5, where the $\mathrm{Cu}$ grains are clearly visible. It also shows the presence of $\mathrm{Cu}$ surface steps, graphene "wrinkles," and the presence of non-uniform dark flakes. The wrinkles associated with the thermal expansion coefficient difference between $\mathrm{Cu}$ and graphene are also found to cross $\mathrm{Cu}$ grain boundaries, indicating that the graphene film is continuous.

In addition to this, the electrical mobility study performed on

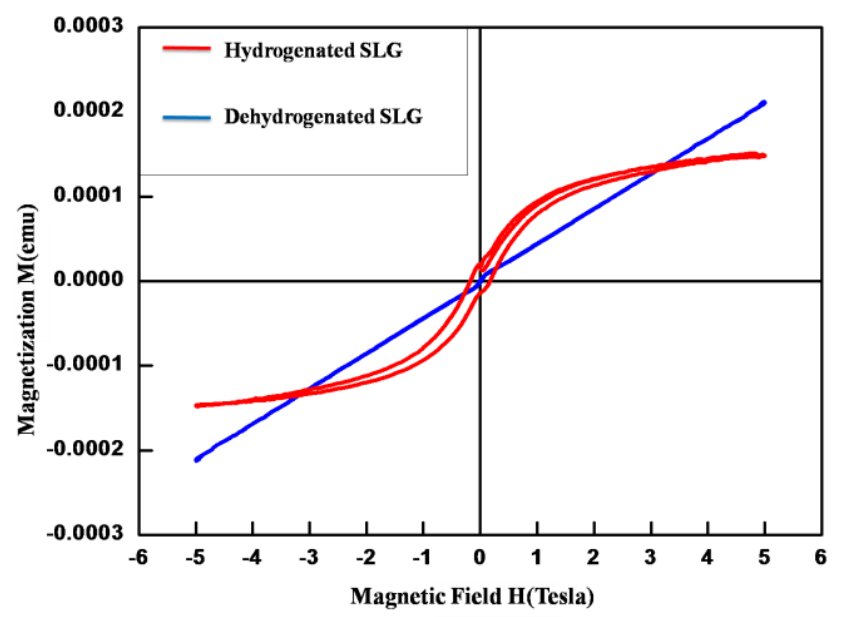

Figure $4 \mathrm{M}-\mathrm{H}$ hysteresis of hydrogenated and dehydrogenated SLG.

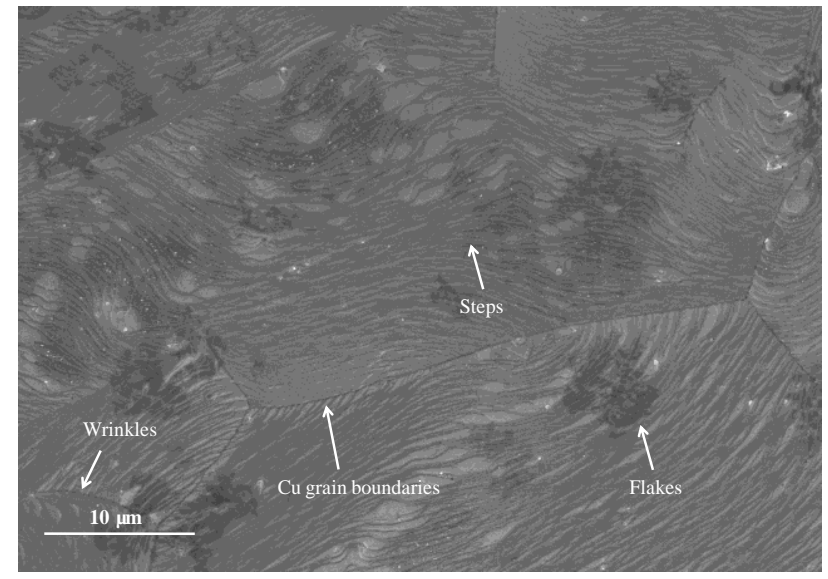

Figure 5 High resolution FESEM image of single layer graphene on $\mathrm{Cu}$ foil grown for $\sim 15 \mathrm{~min}$; showing $\mathrm{Cu}$ grain boundaries and steps, two or three layer flakes and graphene flakes and graphene wrinkles

SLG transferred onto quartz substrate using two probe I-V measurement units also showed an almost complete restoration of the graphitic network after dehydrogenation. Before hydrogenation, the SLG gave a sheet resistance of 150 $\Omega /$ square, which increases to open circuit $39.7 \mathrm{k} \Omega /$ square after hydrogenation. Moreover, the nature of the curve changes from metallic to semiconductor with a finite band gap as is observed in Figure 6. However, this resistance value gets dramatically reduced to $2.4 \mathrm{k} \Omega / \mathrm{square}$ after dehydrogenation.

\section{Conclusions}

In conclusion, SLG was grown on copper foil and transferred onto quartz substrate for hydrogenation using lithium in liquid ammonia with methanol as the source of protons. Several analytical techniques demonstrated a very high degree of hydrogenation. Hydrogenated SLG was refluxed with DDQ in toluene at $110{ }^{\circ} \mathrm{C}$ to facilitate an easy and effective dehydrogenation process. Characterization by FT-IR, UV-Vis, and Raman spectroscopy confirms the dehydrogenation process. Magnetic moment measurement studies reconfirm the reversibility of the dehydrogenation process. Of particular significance is the analysis of the electronic mobility study of the dehydrogenated graphene that shows almost complete restoration of the conjugated SLG structure. The tunable band gap of the 


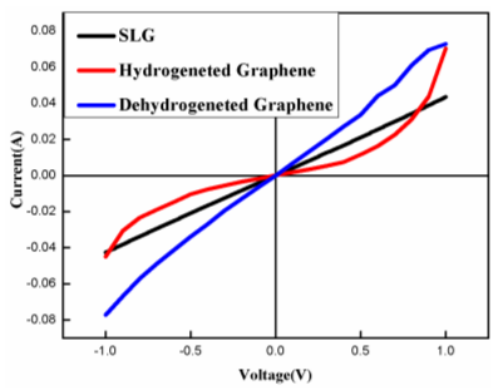

Figure 6 I-V characteristics of SLG, hydrogenated and dehydrogenated SLG

hydrogenated and dehydrogenated SLG obtained during the processes makes it a readily available cheap acceptor material for OPV solar cells.

\section{Acknowledgement}

This work was financially supported by the Science and Engineering Research Board (SERB), Department of Science and Technology (DST), India and gratefully acknowledged. TC appreciates the support of DST Inspire fellowship.

\section{Author Contributions}

Bench scale experiments were performed by PPC and TC. AM supervised the whole process and given intellectual input.

\section{Conflict of Interest}

The authors declare no conflict of interest.

Copyright (C) 2020 Prarthana Paul Chowdhury, Tania Chatterjee, and Arnab Mukherjee. This article is an open access article distributed under the terms and conditions of the Creative Commons Attribution (CC BY) license (http://creativecommons.org/licenses/by/4.0/). The use, distribution or reproduction in other forums is permitted, provided the original author(s) or licensor are credited and that the original publication in this journal is cited, in accordance with accepted academic practice. No use, distribution or reproduction is permitted which does not comply with these terms.

\section{References}

[1] Wallace, P. R. The Band Theory of Graphite. Phys. Rev. 1947, 71, 622-634.

[2] McClure, J. W. Diamagnetism of Graphite. Phys. Rev. 1956, 104, 666-671.

[3] Geim, A. K.; Novoselov, K. S. Diamagnetism of Graphite.Nat. Mater. 2007, 6, 183-191.

[4] Zhang, Y.; Zhang, L.; Zhou, C. Review of Chemical Vapor Deposition of Graphene and Related Applications. Acc. Chem. Res. 2013, 36, 2329-2339.

[5] An, C. J.; Kim, S. J.; Choi, H. O.; Kin, D. W.; Jang, S. W.; Jin, M. L.; Park, J. M.; Choi, J. K.; Jung, H. T. Ultraclean transfer of CVDgrown graphene and its application to flexible organic photovoltaic cells. J. Mater. Chem. A 2014, 2, 20474-20480.

[6] Huang, X.; Yin, Z.; Wu, S.; Qi, X.; He, Q.; Zhang, Q.; Yan, Q.; Boey, F.; Zhang, H.; Jung, H. T. Graphene-Based Materials: Synthesis, Characterization, Properties, and Application. Small 2011, 7, 1876-1902.

[7] Zeng, Z.; Yin, Z.; Huang, X.; Li, H.; He, Q.; Lu, G.; Boey, F.; Zhang, H. Single-Layer Semiconducting Nanosheets: High-Yield Preparation and Device Fabrication. Angew. Chem. Int. Ed Engl. 2011, 50, 11093-11097.

[8] Huang, X.; Qi, X.; Boey, F.; Zhang, H. Graphene-based composites. Chem. Soc. Rev. 2012, 41, 666-686.

[9] Pang, S.; Hernandez, Y.; Feng, X.; Muellen, K. Graphene as
Transparent Electrode Material for Organic Electronics. Adv. Mater 2011, 23, 2779-2795.

[10] Novoselov, K. S.; Geim, A. K.; Morozov, S. V.; Jiang, D.; Katsnelson, M. I.; Grigorieva, I. V.; Dubonos, S. V.; Firsov, A. A. Two-dimensional gas of massless Dirac fermions in graphene. Nature 2015, 438, 197-200.

[11] Heersche, H. B.; Jarillo-Herrero, P.; Oostinga, J. B.; Vandersypen, L. M.; Morpurgo, A. F. Bipolar supercurrent in graphene. Nature 2007, 446, 56-59.

[12] Hirsch,A.; Englert, J. M.; Hauke, F. Wet Chemical Functionalization of Graphene. Acc. Chem. Res. 2013, 46, 87-96.

[13] Criado, A.; Melchionna, M.; Marchesan, S.; Prato, M. The Covalent Functionalization of Graphene on Substrates. Angew. Chem. Int. Ed. Engl. 2015, 54, 10734-10750.

[14] Zhu, J.; Chen, M.; He, Q.; Shao, L.; Wei, S.; Guo, Z. An Overview of the Engineered Graphene Nanostructures and Nanocomposites. RSC Adv. 2013, 3, 22790-22824.

[15] Dreyer, D. R.; Park, S.; Bielawski, C. W.; Ruoff, R. S. The chemistry of graphene oxide. Chem. Soc. Rev. 2010, 39, 228-240.

[16] Whitener, Jr. K. E.; Robinson, J. T.; Sheehan, P. E. Protection from Below: Stabilizing Hydrogenated Graphene Using Graphene Under layers. Langmuir 2017, 33, 13749-13756.

[17] Sofo, J. O.; Chaudhari, A. S.; Barber, G. D. Graphane: A twodimensional hydrocarbon. Phys. Rev. B 2007, 75, 153401-(1-4).

[18] Matis, B. R.; Burgess, J. S.; Bulat, F. A.; Friedman, A. L.; Houston, B. H.; Baldwin, J. W. Surface Doping and Band Gap Tunability in Hydrogenated Graphene. ACS Nano 2012, 6, 17-22.

[19] Jaiswal, M.; Lim, C. H.; Bao, Q.; Toh, C. T.; Loh, K. P.; Ozyilmaz, B. Controlled Hydrogenation of Graphene. ACS Nano 2011, 5, 888-896.

[20] Gao, H.; Wang, L.; Zhao, J.; Ding, F.; Lu, J. Band Gap Tuning of Hydrogenated Graphene: $\mathrm{H}$ Coverage and Configuration Dependence. J. Phys. Chem. C 2011, 115, 3236-3242.

[21] Whitener, Jr. K. E.; Lee, W. K.; Campbell, P. M.; Robinson, J. T.; Sheehan, P. E. Activation of radical addition to graphene by chemical hydrogenation. RSC Adv. 2016, 6, 93356-93362.

[22] Elias, D. C.; Nair, R. R.; Mohiuddin, T. M. G.; Morozov, S. V.; Blake, P.; Halsall, M. P.; Ferrari, A. C.; Boukhvalov, D. W.; Katsnelson, M. I.; Geim, A. K.; Novoselov, K. S. Control of Graphene's Properties by Reversible Hydrogenation: Evidence for Graphane. Science 2019, 323, 610-613.

[23] Yang, Z.; Sun, Y.; Alemany, L. B.; Narayanan, T. N.; Billups, W. E. Birch Reduction of Graphite. Edge and Interior Functionalization by Hydrogen. J. Am. Chem. Soc. 2012, 134, 18689-18694.

[24] Li, X.; Cai, W.; An, J.; Kim, S.; Nah, J.; Yang, D.; Piner, R.; Velamakanni, A.; Jung, I.; Tutuc, E.; Banerjee, S. K.; Colombo, L.; Ruoff, R. S. Large-Area Synthesis of High-Quality and Uniform Graphene Films on Copper Foils. Science 2009, 324, 1312-1314.

[25] Levendorf, M. P.; Ruiz-Vargas, C. S.; Garg, S.; Park, J. Transfer-Free Batch Fabrication of Single Layer Graphene Transistors. Nano Lett.2009, 9, 4479-4483.

[26] Ci, L.; Song, L.; Jin, C.; Jariwala, D.; Wu, D; Li, Y.; Srivastava, A.; Wang, Z. F.; Storr, K.; Balicas, L.; Liu, F.; Ajayan, P. M. Atomic layers of hybridized boron nitride and graphene domains. Nat. Mater. 2010, 9, 430-435.

[27] Song, L.; Ci, L.; Lu, H.; Sorokin, P. B.; Jin, C.; Ni, J.; Kvashnin, A. G.; Kvashnin, D. G.; Lou, J.; Yakobson, B. I.; Ajayan, P. M. Large Scale Growth and Characterization of Atomic Hexagonal Boron Nitride Layers. Nano Lett. 2010, 10, 3209-3215.

[28] Zhou, J.; Wang, Q.; Sun, Q.; Chen, X. S.; Kawazoe, Y.; Jena, P. Ferromagnetism in Semihydrogenated Graphene Sheet. Nano Lett. 2009, 9, 3867-3870.

Received December 4, 2020 Accepted January 21, 2020 